\title{
D-galactose and aluminium chloride induced rat model with cognitive impairments
}

\begin{abstract}
Cognitive impairments and cholinergic dysfunctions have been well reported in old age disorders including Alzheimer's disease (AD). d-galactose (D-gal) has been reported as a senescence agent while aluminium act as a neurotoxic metal, but little is known about their combined effects at different doses. The aim of this study was to establish an animal model with cognitive impairments by comparing the effects of different doses of co-administrated D-gal and aluminium chloride $\left(\mathrm{AlCl}_{3}\right)$. In this study male albino wistar rats were administered with D-gal $60 \mathrm{mg} / \mathrm{kg} . b w t$ intra peritoneally (I.P) injected and $\mathrm{AlCl}_{3}(100,200$, or $300 \mathrm{mg} / \mathrm{kg}$. bwt.) was orally administered once daily for 10 consecutive weeks. Performance of the rats were evaluated through behavioural assessments; Morris water maze (MWM) and open field tests (OFT); histopathological examination was performed on the hippocampus; moreover biochemical measurements of acetylcholinesterase (AChE) and hyperphosphorylated tau protein (p-tau) were examined. The results of this experiment on rats treated with $\mathrm{D}$-gal $60+\mathrm{AlCl}_{3} 200 \mathrm{mg} / \mathrm{kg}$.bwt showed near ideal cognitive impairments. The rats exhibited an obvious memory and learning deficits, marked neuronal loss in hippocampus, showed increase in $\mathrm{AChE}$ activities and high expression of $\mathrm{p}$-tau within the tissues of the brain. This study concludes that D-gal $60+\mathrm{AlCl}_{3} 200 \mathrm{mg} / \mathrm{kg}$.bwt as the ideal dose for mimicking $\mathrm{AD}$ like cognitive impairments in albino wistar rats. It is also crucial to understand the pathogenesis of this neurodegenerative disease and for drug discovery.
\end{abstract}

Keyword: Alzheimer's disease; d-galactose; Aluminium chloride; Cognitive impairment; Neurodegenerative disease; Dementia; Acetylcholinesterase; Neurofibrillary tangles; Senile plaques; Beta amyloid; Alzheimerogenic chemicals; Learning dysfunction; Hyperphosphorylated tau protein; Neurotoxins; Morris water maze; Hippocampus; Non-transgenic; Cornus ammonis; Open field test; Western blot 